\title{
Mycobacterium chelonae Cutaneous Infection: A Challenge for an Internist
}

\author{
Margarida Gaudêncio, André Carvalho, Maria Inês Bertão, Ivo Barreiro, Maria Isabel Bessa, Abílio Gonçalves \\ Department of Internal Medicine, Hospital Distrital da Figueira da Foz, EPE, Figueira da Foz, Portugal
}

Received: 18/10/2021

Accepted: $24 / 10 / 2021$

Published: 15/11/2021

How to cite this article: Gaudêncio M, Carvalho A, Bertão MI, Barreiro I, Bessa MI, Gonçalves A. Mycobacterium chelonae cutaneous infection: a challenge for an internist. EJCRIM 2021;8: doi:10.12890/2021_003013.

Conflicts of Interests: The authors declare there are no competing interests. This case was presented at the 11th International Congress of Internal Medicine as an e-poster.

This article is licensed under a Commons Attribution Non-Commercial 4.0 License

\section{ABSTRACT}

Cutaneous infections caused by the Mycobacterium chelonae complex show a heterogeneous clinical presentation, which varies according to the patient's immune status. Most standard antimycobacterials have no effect against these species. Clarithromycin alone was shown to provide adequate treatment, although resistance has been reported. Consequently, the literature supports multi-drug therapy to combat resistant strains. Here, we describe the case of a 59-year-old man under systemic immunosuppressive therapy who developed cutaneous lesions whose evolution was highly suggestive of atypical infection.

\section{LEARNING POINTS}

- Mycobacterium chelonae is a ubiquitous species of mycobacteria found in the environment; cutaneous infections by the M. chelonae complex show a heterogeneous clinical presentation.

- The combination of linezolid and clarithromycin has been used in some cases, with rapid clinical resolution and less or no relapse observed after long-term follow-up.

- Internal Medicine is the appropriate speciality to treat particularly complex or multisystem diseases, such as infections in immunocompromised patients.

\section{KEYWORDS}

Mycobacterium chelonae, cutaneous infection, immunosuppression, atypical specimens

\section{INTRODUCTION}

Mycobacterium chelonae is a non-tuberculous, rapidly growing species of mycobacteria that is often grouped together with Mycobacterium fortuitum and abscessus due to their similar clinical and metabolic features ${ }^{[1]}$. It is found in soil, dust and natural water supplies ${ }^{[1]}$. Infection typically follows incidental environmental inoculation ${ }^{[1]}$.

The cutaneous manifestations vary according to the patient's immune status ${ }^{[2]}$. Localized infections such as cellulitis and abscesses are commonly seen in immunocompetent patients, while disseminated skin disease occurs in immunosuppressed patients (organ transplantation, chemotherapy, autoimmune disorders, long-term steroid therapy or biologic therapies) ${ }^{[2]}$.

In disseminated disease, the limbs are predominantly involved and show different types of lesions such as pustules, nodules, abscesses or ulcers, commonly with a sporotrichoid pattern ${ }^{[3,4]}$. Other less common presentations reported in the literature include osteomyelitis, pneumonitis, lymphadenitis, corneal ulcers, endocarditis and catheter-related infections ${ }^{[5]}$.

Histopathological findings are non-specific and tend to include a granulomatous inflammatory cell infiltrate with abundant neutrophils ${ }^{[6]}$. Dermal or subcutaneous abscesses, acute or chronic panniculitis or a non-specific chronic inflammation can be found ${ }^{[6]}$.

The authors describe a very unusual case of M. chelonae infection with severe cutaneous involvement after long-term immunosuppressive therapy. 


\section{CASE DESCRIPTION}

A 59-year-old man diagnosed with sarcoidosis in 1978 was under systemic immunosuppressive therapy with azathioprine $100 \mathrm{mg} / \mathrm{day}$ and methylprednisolone $8 \mathrm{mg} /$ day. He also had a history of chronic obstructive pulmonary disease, a pulmonary embolism in 2019 (negative study for thrombophilia and antiphospholipid syndrome), obstructive sleep apnoea and respiratory failure requiring nocturnal non-invasive ventilation and daytime oxygen therapy. The patient did not have any tattoos.

The patient presented a 2-week history of multiple painful, erythematous nodules distributed over the posterior right arm (Fig. 1A). Cutaneous biopsy showed abscess formation and no granulomatous infiltrate.

The first cultures identified Turicella otitidis and the patient started cefuroxime. However, he did not show any improvement and so cultures were repeated, revealing the presence of Nocardia and Actinomyces.

After 3 months of therapy with trimethoprim/sulfamethoxazole, the patient developed more skin lesions (erythematous papules and nodules, pustular lesions and haemorrhagic crusts) distributed over the limbs, face, trunk and abdomen (Fig. 1B).

About 2-3 months after the first appearance of the skin lesions, the patient experienced sporadic episodes of rectal bleeding. A study of the digestive tract was requested, but the patient postponed investigation. However, he experienced frequent episodes of rectal bleeding and anaemia (haemoglobin 12.3-12.7 g/dl) associated with weight loss (10 kg in 1 month). So, 5 months after symptom onset, an endoscopy and colonoscopy were performed. Thoraco-abdominopelvic tomography showed known diffuse emphysematous changes in pulmonary parenchyma and several calcified hilar and mediastinal ganglion cysts, as well as absence of metastasis.

Rectal adenocarcinoma was diagnosed and the patient was submitted to radiotherapy before abdominoperineal resection.

The patient was later hospitalized due to a cytomegalovirus infection and for intravenous antibiotics (Fig. 1C).

Skin cultures were repeated and research laboratory support was requested for molecular analysis, which revealed $M$. chelonae (susceptible to amikacin, ciprofloxacin, linezolid and clarithromycin).

The patient started a 12-month linezolid and clarithromycin regimen. Currently, he has complete 9 months of therapy with progressive improvement in skin lesions (Fig. 1D).

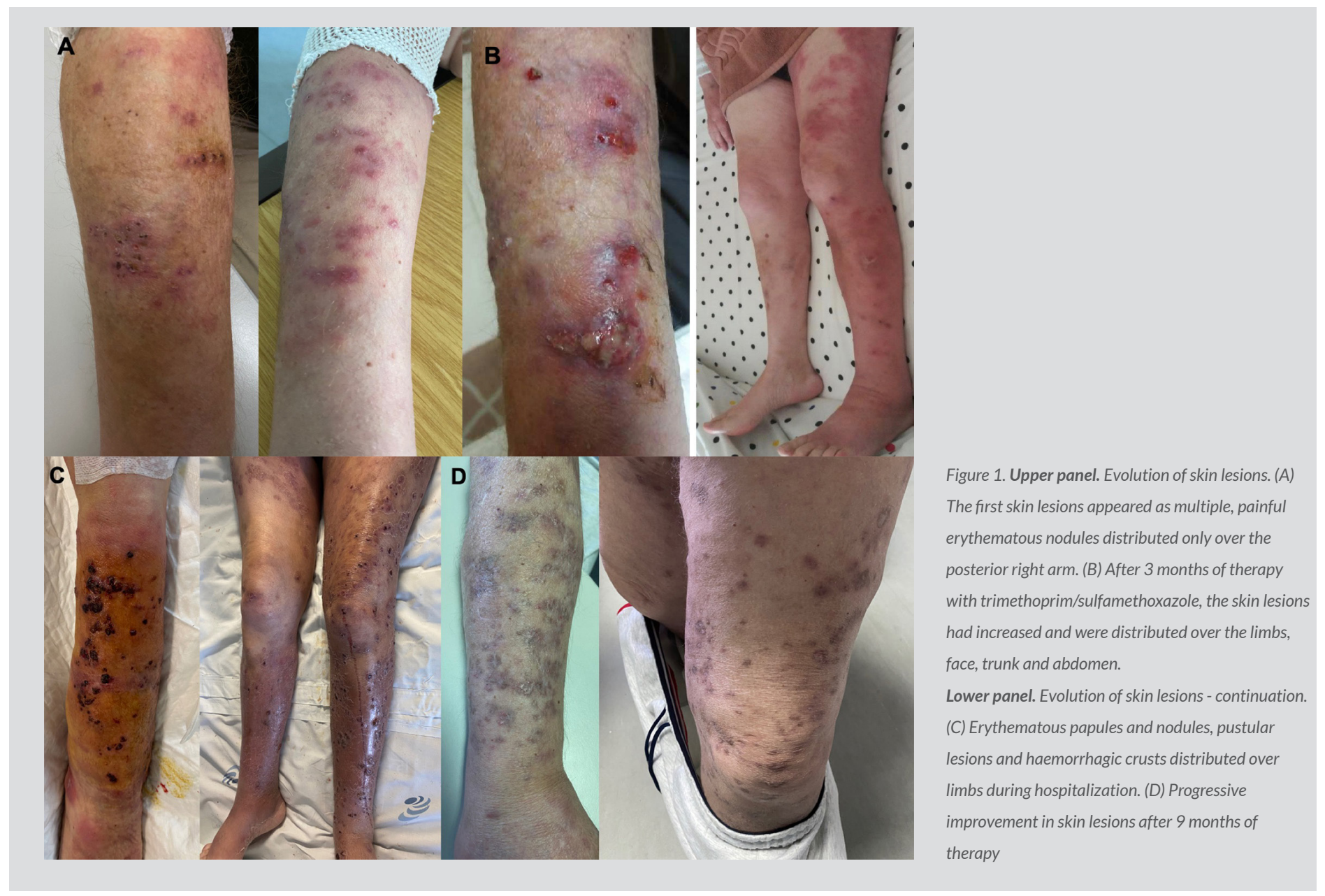




\section{DISCUSSION}

Skin, bone and soft tissue diseases are the most frequent clinical manifestations of $M$. chelonae infection ${ }^{[7]}$. Our case is notable due to the numerous pustules, nodules, abscesses and haemorrhagic crusts distributed over the limbs, face, trunk and abdomen, showing a sporotrichoid pattern.

Skin infections have been reported following surgery, acupuncture, mesotherapy and tattooing ${ }^{[8]}$. In the case of tattoos, the infections affect areas coloured grey, as non-sterile water is added to black ink [8]. In this case, the patient did not have any tattoos, so inoculation of this agent may have occurred during contact with soil and exotic plants. The fact that the patient was immunosuppressed also contributed to infection.

Most standard antimycobacterials such as ethambutol, pyrazinamide and isoniazid, have no effect against these species ${ }^{[9]}$. Clarithromycin alone was shown to provide adequate treatment, although resistance has been reported ${ }^{[10]}$. Resistance to other antimicrobials such as erythromycin, ciprofloxacin, ofloxacin and sulfamethoxazole, has also been described ${ }^{[11]}$. The literature supports multi-drug therapy to avoid selecting resistant strains and a prolonged therapy period in order to prevent relapse ${ }^{[12]}$.

Treatment for this type of infection has not yet been standardized ${ }^{[13]}$. Guidelines published by the American Thoracic Society on nontuberculous mycobacterial diseases in 2007 recommend a minimum of 4-6 months of treatment with combination antibiotic therapy for cutaneous infections ${ }^{[13]}$. However, there are differences between treatment for immunocompetent and immunocompromised patients ${ }^{[2]}$. We report this case because of its striking and unusual clinical presentation, which provides agood example of the role of immunosuppression as a major risk factor for non-tuberculous mycobacterial infection.

This case also highlights the importance of treating these types of cases with an extended course of appropriate antibiotics, particularly in immunocompromised patients. Furthermore, there is clearly a need for treatment guidelines for M. chelonae cutaneous infections.

In conclusion, with this case, the authors hope to alert physicians to consider M. chelonae infection in cases of intractable skin lesions and to collect specimens and culture pathogens, repeatedly if necessary, as we did. We isolated different atypical agents at an early stage, but due to the lack of therapeutic response, continued our search until we identified the species responsible.

\section{REFERENCES}

1. Kullavanijaya P. Atypical mycobacterial cutaneous infection. Clin Dermatol 1999;17:153-158; discussion 105-106.

2. Halpern J, Biswas A, Cadwgan A, Tan BB. Disseminated cutaneous Mycobacterium chelonae infection in an immunocompetent host. Clin Exp Dermatol 2010;35:269-271.

3. Chung WK, Kim MS, Kim CH, Lee MW, Choi JH, Moon KC, et al. Cutaneous Mycobacterium chelonae infection presenting as symmetrical plaques on both shins in an immunocompetent patient. Acta Derm Venereol 2009;89:663-664.

4. Haas SR, Hodge MB, Duncan RA. Cushing's syndrome presenting as disseminated cutaneous Mycobacterium chelonae infection. Clin Infect Dis 2001;33:e51-e53.

5. Kane CL, Vincent AL, Greene JN, Sandin RL. Disseminated cutaneous Mycobacterium chelonae infection. Cancer Control 2000;7:181-184.

6. Bartralot R, Pujol RM, García-Patos V, Sitjas D, Martín-Casabona N, Coll P, et al. Cutaneous infections due to nontuberculous mycobacteria: histopathological review of 28 cases. Comparative study between lesions observed in immunosuppressed patients and normal hosts. J Cutan Pathol 2000;27(3):124-129.

7. Phillips K, Blackford S, Berry N. Disseminated cutaneous Mycobacterium chelonae infection with multidrug resistance in a patient with panuveitis. Clin Exp Dermatol 2008;33(3):256-258

8. Lapresta A, Pérez C, García-Almagro D. Lesiones faciales tras tatuajes. Actas Dermosifiliogr 2010;101:889-890.

9. Iseman M. Group IV: rapid growing mycobacteria. In: Yu VL, Merigan TC, Barriere SL, editors. Antimicrobial therapy and vaccines. Philadelphia: William \& Wilkins; 1999, pp. 494-497.

10. Morihara K, Takenaka H, Morihara T, Katoh N. Cutaneous Mycobacterium chelonae infection successfully treated with faropenem. J Dermatol 2011;38:211-213.

11. Wallace RJ Jr, Brown BA, Onyi GO. Skin, soft tissue, and bone infections due to Mycobacterium chelonae chelonae: importance of prior corticosteroid therapy, frequency of disseminated infections, and resistance to oral antimicrobials other than clarithromycin. J Infect Dis 1992;166:405-412.

12. Kullavanijaya P, Rattana-Apiromyakij N, Sukonthapirom-Napattalung P, Sirimachand S, Duangdeeden I. Disseminated Mycobacterium chelonae cutaneous infection: recalcitrant to combined antibiotic therapy. J Dermatol 2003;30:485-491.

13. Griffith DE, Aksamit T, Brown-Elliott BA, Catanzaro A, Daley C, Gordin F, et al. An official ATS/IDSA statement: diagnosis, treatment, and prevention of nontuberculous mycobacterial diseases. Am J Respir Crit Care Med 2007;175(4):367-416. 